\title{
Advances in Relativistic Fluid Dynamics, Observables, and Ap- plications - In Memoriam Walter Greiner
}

\author{
L. P. Csernai ${ }^{1, a}$ \\ ${ }^{1}$ Dept. of Physics and Technology, Univ. of Bergen, Norway
}

\begin{abstract}
Walter Greiner was one of the first physicists using Relativistic Fluid Dynamics for High Energy Nuclear Reactions. The present Inertial Confinement Fusion research and development is hindered by hydrodynamic instabilities, occurring at the intense compression of the target fuel by energetic laser beams. The suggested method combines recent advances in two fields: detonations in relativistic fluid dynamics and radiative energy deposition by plasmonic nano-shells. The compression of the target can be negligible and a laser pulse achieves rapid volume ignition, which is as short as the penetration time of the light across the pellet. The reflectivity of the target can be made negligible, and the absorptivity can be increased by one or two orders of magnitude using plasmonic nanoshells embedded in the target fuel. Thus, higher ignition temperature can be achieved with modest compression. The short light pulse can heat most of the interior of the target to the ignition temperature simultaneously. This prevents the development of any kind of instability, which would prevent complete ignition or transition of the target.
\end{abstract}

\section{Introduction}

Fusion energy production is a long-term goal of human society, which provides massive energy production with limited use of space, smallest amount of fuel and negligible waste! We address one of the two major directions of this research and development the Inertial Confinement Fusion (ICF). This research reached a stage where the energy production is verified at the National Ignition Facility (NIF) in the Lawrence Livermore National Lab (LLNL) in USA, but the progress is slow and not satisfactory. We envisage a major change in the direction of this research with a fundamentally different approach. These are based on two novel discoveries in fundamental research: (1) Relativistic Fluid Dynamics is well studied in Relativistic Heavy Ion Reactions, including the burning (hadronization) of Quark Gluon Plasma (QGP), and in (2) Nano plasmonics, where light absorption is increased by one or two orders of magnitude with embedding resonant golden nano-shells into the target material.

The envisaged breakthrough is to achieve effective, simultaneous volume ignition in the Deuterium (D) Tritium (T) target fuel, without instabilities. The major obstacle of present technological development that Rayleigh-Taylor (RT) instabilities block the full ignition of the target. We aim for theoretical studies of the new methodology, and experimental verification of some of the new fundamental features of the method.

ae-mail: laszlo.csernai@uib.no 
The primary objective is to develop the theoretical foundation for achieving ignition of the Deuterium-Tritium (DT) ignition fuel in Inertial Confinement Fusion, using a linear configuration, through implementation of concepts from Relativistic Fluid Dynamics (RFD) and Nano-Technology. These methods will also be extended to heavy ion induced fusion.

The basic methodical innovation is to use relativistic fluid dynamics (RFD) instead of classical fluid dynamics. RFD has new qualitative features especially in the field of relativistic detonations. Presently in the field of ICF research and all standard fluid dynamical applications, these are not known. Up to now, this methodology is only used in ultra-relativistic heavy ion physics. In this field, the energy release of the hadronizing Quark Gluon Plasma is exactly an ultra-relativistic, time-like "detonation". On the Earth up to now, there were no phenomena where this type of process occurred.

Thus, we are going to use this methodology, Relativistic Fluid Dynamics, to develop a new technology. This interdisciplinary approach is a spin-off of the high-energy fundamental research on the Earth.

We propose to study two possible pellet ignition scenarios:

1.) Linear ignition: Heating by laser light absorption is possible without compression. Here, a 3dimensional geometry has no advantages. Linear irradiation of a flat target by two lasers from opposite sides is a much simpler and easier approach, less demanding and much cheaper than a spherical compression facility as NIF. Universities and national labs of smaller countries can therefore even use such a set-up. In addition, the theoretical treatment is simpler and the correspondence between theory and experiment is under much better control. The present work in this field considers only spherical targets with an array of multiple lasers. Using relativistic fluid dynamics, we argue that the target only needs to be heated uniformly in order to reach ignition. Therefore, a configuration using a flat-surface target and two lasers can reach ignition conditions. An important first step in our project plan will therefore be to modify existing equations from spherical to linear symmetry. This is the 1st significant task and milestone of the project.

2.) Ignition through heavy ion beams: Ignition of the fuel target can also be induced by heavy ion beams instead of laser light. The advantage of ion beams is that we are able to increase the spatial precision of the energy deposition in the target due to the well-known features of the Bragg peak. This technology is already used, for example in cancer radiation therapy. This alternative will also be studied in dynamical models. The present project plan is to study and improve the present ICF technology using the presented methodology and results of fundamental research.

\section{Novelty and foundational character}

Our team has extended activity in Relativistic Heavy Ion theory, and RFD. It is of our opinion that relativistic fluid dynamics, a part of CERN heavy ion reactions, can and must be used for all high temperature phenomena. It provides a possibility to avoid the Rayleigh-Taylor instabilities, which at present are the most serious obstacles in ICF. At the Lawrence Livermore National Laboratory in the National Ignition Facility, the Deuterium-Tritium fuel target is compressed by tremendous pressure, but not uniformly! This is due to the RT instability. The compression and thus the adiabatic temperature increase is not uniform in the target and only a very small fraction of the fuel pellet is ignited (see the high-density, red, regions of the pellet).

In the field of high-energy heavy ion physics, important and very relevant discoveries were made in the last decades. First of all the correct description of the relativistic development were developed in 1987 [, 2] in contrast to the previous work of A. Taub, dating back to 1948, which missed the possibility of "time-like" detonations due to a sign oversight. This early result was taken over to all textbooks (including e.g. the book of Landau \& Lifsitz), and the time-like detonations are still 
today considered to be un-physical in most fields of research and technology, except in relativistic heavy ion research. Here it became standard material in textbooks (e.g. L.P. Csernai: Introduction to Relativistic Heavy Ion Collisions (Wiley, 1994)). Since then the time development of relativistic detonation and burning of Quark Gluon Plasma, is always described as a time-like detonation, i.e. simultaneous burning in the whole volume at the same time. In such a situation, the development of any mechanical, RT instability is excluded. This possibility was pointed out recently in ref. [3] for use in ICF.

Contrary to this partial ignition the energy of the produced fused fuel exceeded the laser energy that was absorbed by the target DT pellet (although this was only a small fraction of the total beam energy of the laser shot used to achieve ignition).

The basic methodical innovation of the project is to use relativistic fluid dynamics (RFD) instead of classical fluid dynamics. RFD has new qualitative features especially in the field of relativistic detonations. Presently in the field of ICF research and all standard fluid dynamical applications, these are not known. Up to now, this methodology is only used in ultra-relativistic heavy ion physics. In this field, the energy release of the hadronizing Quark Gluon Plasma (QGP) is exactly an ultrarelativistic, time-like "detonation". On the Earth up to now, there were no industrial or technological phenomena where this type of process occurred. Thus, the theoretical description was developed for "slow" non-relativistic processes only.

The time development of the burning (or hadronization) of QGP in the final expansion stage of two ultra-relativistic heavy ion reactions at the CERN Large Hadron Collider has the following tendency. Initially the QGP matter slowly expands and burns for 10-12 fm/c time and then the whole volume of the target burns at the same time. The points of this burning front are not in causal connection, thus no mechanical instabilities can develop due to their interaction. The burning (hadronization) profile for two colliding energies, 5.5 and $39 \mathrm{TeV} /$ nucleon, are showing the same features [4].

We are using this methodology, Relativistic Fluid Dynamics, in present day developing technology. This is an interdisciplinary approach and a spin-off of the highest energy fundamental research on the Earth.

As described above in the present status of the ICF research, the present standard in this research is to achieve ignition by adiabatic compression and adiabatic heating with non-relativistic dynamics. This approach is hindered primarily by the occurrence of Rayleigh-Taylor instability, which reduces the original ignition volume to less than $10 \%$ of the target.

The present proposal goes into an opposite direction: it does not aim for high pressure and compression; it uses heating by light absorption and rapid heating in the fuel target without significant compression, but at relativistic speed, i.e. with a much shorter, (by three orders of magnitude) and equally energetic laser pulse.

In addition, it is absolutely new that we use methods taken from Nano-Technology to regulate light absorption, as described below, to be able to reach simultaneous, whole volume ignition.

These two features are new concepts in this field, just recently patented and never used before.

Detailed project considerations: The present project plan is to study and improve the present ICF technology using the presented methodology and fundamental results. It was shown in ref. [3] that this is possible but in the presented example calculation, the time-like detonation was extended to the half of the DT target radius, thus only to about $12 \%$ of the target. This is not sufficient to achieve a full volume simultaneous ignition.

In a recent Patent [5] this problem is addressed and a solution was presented: The target is not compressed but heated up with a short light impulse of laser(s). With methods from Nano-Technology, one can change the light absorptivity of the DT target by imbedding Golden Nano-shells into the target and avoiding the external Golden "Hohlraum" as well as the surface ablator layer. 
The light propagates form the edge of the target inwards. The middle of the target is reached later by the laser light than the outside edge. If we want to achieve a simultaneous volume ignition, we have to heat up the middle of the target more effectively than the edges. One can achieve this by embedding an increased density of golden nano-shells in the middle domain of the target. We can achieve this way that the edges and the centre reach the ignition temperature at the same time. According to [5] one can reach simultaneous ignition up to a radius of $90 \%$ of the pellet radius, so one can reach simultaneous volume ignition in $73 \%$ of the target volume (see figure 1).

The present work in this field considers only spherical targets with an array of multiple lasers. Using relativistic fluid dynamics, we argue that the target only needs to be heated uniformly in order to reach ignition. Therefore, a configuration using a flat surface target and two lasers can be made to reach ignition conditions. An important first step in our project plan will therefore be to modify existing equations from spherical to linear symmetry.

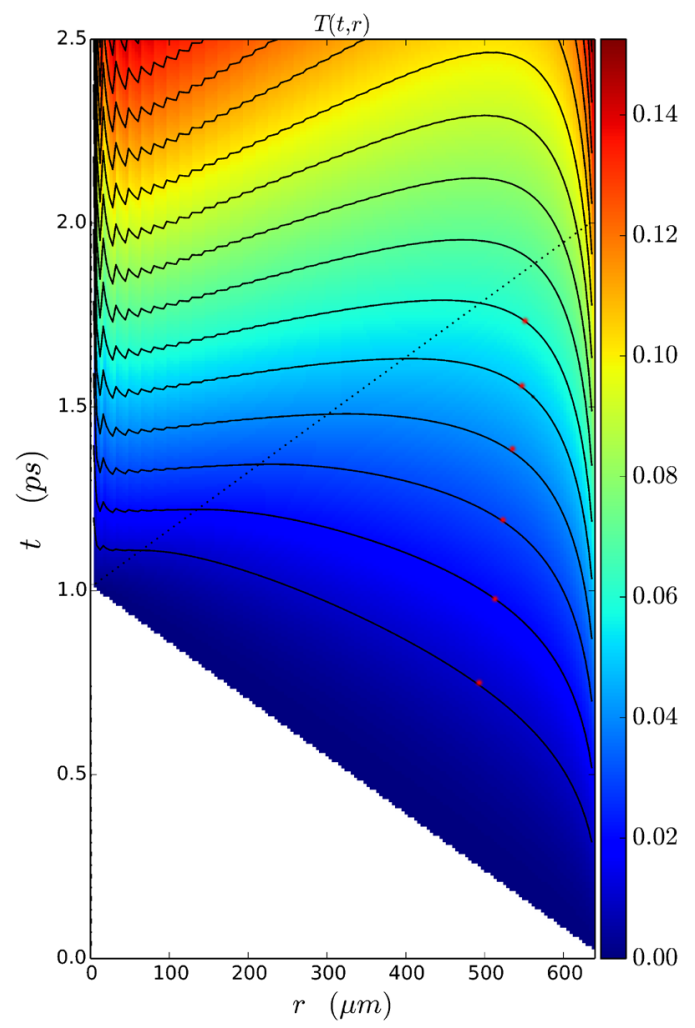

Figure 1. The temperature, $T(t, r)$, increase in the fuel target as a function of time $(t)$ and radius, in units of $(\mathrm{MeV})$, where the constant temperature contour lines are indicated. The heating starts on the light-cone. By the 4th or 5th contour line (the ignition temperature) is reached in $90 \%$ of the radius simultaneously, which corresponds to $73 \%$ of the total fuel volume. The compression of the target is neglected.

Additional advantages of the new concept: The ignition attempts based on adiabatic compression have an advantage in 3-dimensional geometry, because the density and temperature goes with the third power of the linear compression.

Arguments for the foundational nature of the breakthrough, in the sense that, if achieved, it would establish an essential basis for a new line of scientific and/or technological research and its future uses, not currently anticipated.

The two novel aspects used in the proposal originate from very recent fundamental research, never used in modern technology or research before. Its use in technology and development is new, and never tried or even suggested before for any field of modern technology. It goes against present 
intentions and practices of ICF research. It is risky, but may lead to a full breakthrough with more modest expenses than the present research attempts.

\section{Research methodology}

Linear ignition: In case of heating by light absorption without compression, the 3-dimensional geometry has no advantages. At the same time, to realize the linear irradiation of a flat target by two lasers from the opposite side a much more simple and easier, which can be done even with much smaller expenses in smaller countries. In addition, the theoretical treatment is also simpler and the correspondence between theory and experiment is under much better control. This is the first significant task and milestone of the project.

Ignition by heavy ion beams: Ignition of the fuel target can also be induced using heavy ion beams instead of light lasers. The advantage of ion beams is that we are able to increase the spatial precision of the energy deposition in the target due to the well-known features of the Bragg peak. This technology is already used in for example cancer research. For ignition conditions to be reached using such ion beam configurations, we need beams with a 10-picosecond bunch length. Currently, the bunch length at the CERN CMS detector is 70 nanosecond, i.e. about 4 orders of magnitude longer. It is not impossible to improve this. This is the second significant task and milestone of the project.

Both these two development goals require substantial theoretical and computational effort. Furthermore, some aspects of the new materials, e.g. DT fuel may have to be tested experimentally. For the experimental tests Hungary and Romania are part of the European Light Infrastructure (ELI) project, with good connections to our Partner institutions in these countries.

The need of eventual pre-compression of the fuel target is not clarified yet. This may achieve a better Lawson criterion. In this work the lack of information on material properties under the proposed circumstances may occur. Our close contacts to experimental laser and accelerator research centres will be able to surmount these difficulties. More extensive experimental effort will be necessary to solve this problem.

\section{References}

[1] L. P. Csernai, S. F. Spinnangr, S. Velle, Quantitative Assessment of Increasing Complexity, Physica A 473, 363-376 (2017).

[2] L. P. Csernai, Detonation on a timelike front for relativistic systems, Zh. Eksp. Teor. Fiz. 92, 379-386 (1987).

[3] L. P. Csernai, D. D. Strottman, Volume ignition via time-like detonation in pellet fusion, Laser and Particle Beams 33, 279-282 (2015).

[4] L.P. Csernai: Introduction to Relativistic Heavy Ion Collisions, (John Wiley \& Sons, Cichester, England, 1994); U.W. Heinz and P.F. Kolb, Phys. Lett. B 542, 216 (2002); R. Chatterjee, et al., Phys. Rev. Lett. 96, 202302 (2006); E. Molnar, et al., J. Phys. G 34, 1901 (2007); E. Frodemann, et al., J.Phys. G 34, 2249 (2007); L.P. Csernai, et al., J.Phys. G 36, 064032 (2009); S. Floerchinger, and U.A. Wiedemann, Phys. Rev. C 89, 034914 (2014); N. Armesto, et al., Nucl. Phys. A 931, 1163 (2014).

[5] L. P. Csernai, N. Kroo and I. Papp, Procedure to improve the stability and efficiency of laser-fusion by nano-plasmonics method, Patent \# P1700278/3 (2017) of the Hungarian Intellectual Property Office. 